\title{
Further Studies on the Polytene Chromosomes of Stylonychia mytilus (Ciliata, Hypotrichia)
}

\author{
María A. Jareño \\ Centro de Investigaciones Biológicas, C S.I.C., Velázquez 144, \\ Madrid 28006, Spain
}

Accepted May 7, 1993

Hypotrichous ciliates have two kinds of nuclei, the macronucleus and the micronucleus. The macronucleus is considered the somatic or vegetative nucleus as it is responsible for the major part of the RNA synthesis during the vegetative life of the ciliate. The micronucleus is diploid and principally generative. In sexual processes such as the conjugation, the macronucleus disappears. After a series of divisions of the micronucleus, one product of the synkarion division gives rise to a new macronucleus. Polytene chromosomes are transitorily formed during the macronuclear anlage development.

The discovery of polytene chromosomes in ciliates was simultaneously made in Stylonychia sp. (Ammzrmann 1964) and Nyctotherus cordiformis (Golikowa 1964), and soon afterwards they werc observed in various ciliates (Alonso and Pérez 1965, Radzikowski 1967, Rao 1968).

In spite of the time that passed, studies on the morphology of these chromosomes have been scarce and some morphological characteristics are still in discussion. One of these is the existence (Jareño 1974) or not (Ammermann 1971) of somatic pairing during early development of the polytene chromosomes in species of the Stylonychia mytilus complex (Wirnsberger et al. 1986). A little more attention has been focused on the question concerning possible roles of the macronuclear anlage (Alonso and Jareño 1974, Gaude 1981, Golikowa and Nilowa 1984, Jareño and Alonso 1973, Sapra and Dass 1970) and on the morphological and molecular characteristics of the macronuclei (revs. Raikov 1982, Jeon (ed.) 1986, Gall (ed.) 1986).

The difficulty of observation of these chromosomes due to their thinness and lability, and the scanty information in literature, leaves the problem of somatic pairing in Stylonychia mytilus open. We show now chromosomal loops that could be the demonstration of the occurrence of somatic pairing of homologous chromosomes during the polytene chromosome development in this species.

\section{Materials and methods}

The strain of the Stylonychia mytilus utilized for studies of polytene chromosomes was isolated from water of a brook near Madrid. It was cultivated in soil medium at $21^{\circ} \mathrm{C}$. and fed with Chlorogonium sp. Complementary mating types of this species were used to obtain exconjugants. Some micrographs are from fresh unfixed and unstained preparations, others were made after staining with acetic orcein as previously described (Jareño 1976).

\section{Results}

The general morphology of the polytene chromosomes during macronuclear anlage development in Stylonychia mytilus was described earlier (Jareño et al. 1972), but for clearer understanding a brief description of the process seems necessary here. 

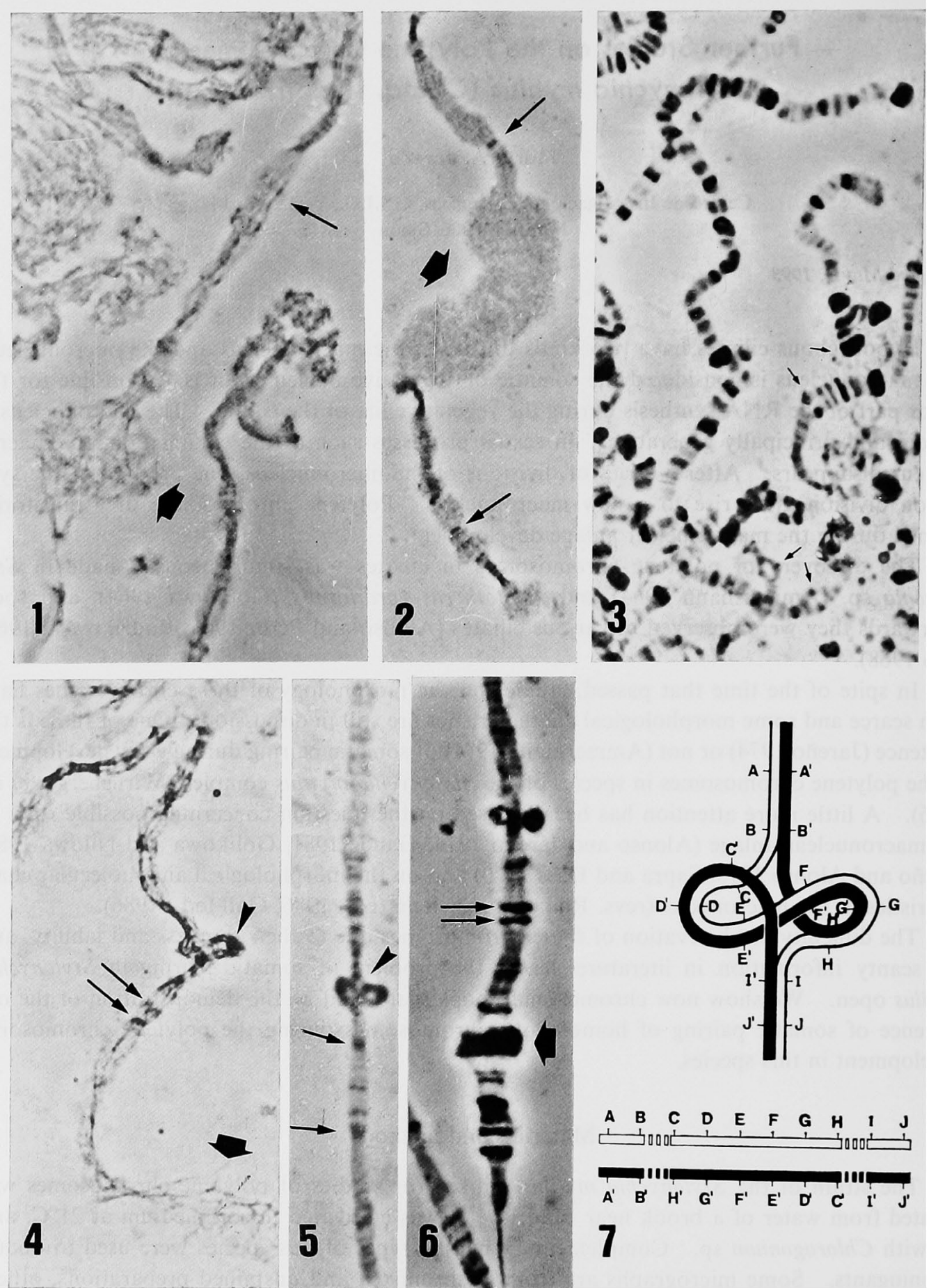

7

Figs. 1-3. Micrographs of the polytene chromosomes of Stylonychia mytilus 1, Chromosomes in stage 2, after acetic orcein staining. 2. Chromosome in stage 3, in fresh preparation. 3. Chromosomes in stage 4 , in fresh preparation. Figs. 4 and 6 . Micrographs of chromosomal loops in S. mytilus. Fig. 4. Chromosomes in stage 2. Acetic orcein. Fig. 5. Chromosomes in stage 3, in fresh preparation. Fig. 6. Chromosome in stage 4, in fresh preparation. Fig. 7. Schematic drawing of a chromosomal loop. Explanation in the text. 
These chromosomes develop from approximately $16 \mathrm{hr}$ to $38 \mathrm{hr}$ after separation of the conjugants. The process is continuous and intermediate images are observed. Nevertheless, four stages for studying these chromosomes can be established. 1. Chromosomes in early macronuclear anlage are spiralized and condensed. 2. Approximately at $26 \mathrm{hr}$ the breaking (in the preparation) of the macronuclear anlage envelope leaves free large diffuse polytene chromosomes which are difficult to observe unstained. Some segments of these chromosomes become parallel filaments (Fig. 1, thin arrow) and others spread filaments (Fig. 1, big arrow) after aceto-orcein staining. 3. Chromosomes at this stage have alternating diffuse and condensed bands. Some of them show more or less enlarged regions (Fig. 2, big arrow), which make their identification possible among the other chromosomes of the macronuclear anlage. Chromosomes in this stage are more easily observable in fresh preparations although the contrast with the medium is not good. They are very delicate, especially in the earlier stages, becoming more and more condensed during development. 4. Chromosomes showing condensed bands and heterochromatic blocks (Fig. 3). In this figure some small longitudinal splits are observed (arrowhead). The polytene chromosomes in this phase are very compact and relatively easy to handle and obtain micrographs.

Two last phases in the macronuclear developement are observed. After stage four, when the macronuclear envelope is mechanically broken, no chromosomes but many small pieces (that correspond to isolated bands and heterochromatic blocks) are seen. Soon afterwards the contents of the macronuclear anlage stay united even when the macronuclear envelope is mechanically broken. Finally, the macronucleus of the exconjugant begins the first division.

Fig. 4 shows a polytene chromosome in stage 2. A chromosomal loop (arrowhead) is clearly visible after acetoorcein staining. Some bands are marked by arrows. A region of extended filaments has been marked by a big arrow. Fig. 5 shows a loop similar to that of the preceding figure but in a chromosome more advanced in development (stage 3), and in a fresh preparation micrograph. Bands are clearly observed along the chromosome (some of them marked by arrows) but not in the loop where bands have a very condensed appearance. Homologous chromosomes are not fused in a small section beyond the inversion (arrowhead). Fig. 6 shows a chromosome in stage 4. A large heterochromatic block is marked by a big arrow.

Fig. 7 is a diagrammatic interpretation of the chromosomal loop. The schematic design shows a loop with a paired intercalary segment. Only the middle of the inverted section of the loop is fused. The hypothetical linear location of the bands in one homologue chromosome and the inverse location in the other is also represented. The inverted region has been limited by discontinuous traces.

Chromosomal deficiencies such as the loops, longitudinal splits and small asymmetrical loops have been frequently observed in polytene chromosomes of exconjugants coming from old mating types that had remained in the laboratory for about three years.

\section{Discussion}

This paper shows qualitative results due to the actual variability of the size, especially the width, of these chromosomes discussed previously (Jareño 1990). The polytene chromosomes of ciliates are very delicate in stages that have been numbered 2 and 3 . For this reason, only macronuclear anlages with chromosomes in stage 4 have been shown in the literature (Raikov 1982), and no individual chromosomes have been described except in Stylonychia mytilus (Jareño et al. 1972, Jareño 1990). Thus comparative studies of polytene chromosomes in ciliates are not possible at the moment. It is necessary to look at the first papers of Diptera polytene chromosomes to compare their morphology. 
The movements of chromosomes and the mechanisms of pairing in the presence of homologues showing structural differences must necessarily be a complicated process. In addition many of the numerous chromosomes of Stylonychia mytilus are long and compressed into a small space.

The observation of the chromosomal loop in different stages and the bands marked in the paired arms leads to the thought that this structural deficiency appears in the same chromosome.

Separate bands are not observed in the loop. In spite of this we think that the structure studied in this paper represents an inversion where the loop was caught between the single arms. This interpretation has been illustrated in the schematic design of Fig. 7. Similar loops demonstrative of chromosomal inversions have been observed in natural populations of Sciaridae (Brito da Cunha et al. 1973) and Drosophila (Jha and Rahman 1973). Chromosomal inversions were present in most of the larvae collected and maintained in laboratory cultures, according to these authors. The image of the loop shown in Results could be a type of false interlocking found commonly in Diptera chromosomal inversions when one chromosome is inserted into a loop of the inversion.

Other interpretations are also possible e.g., one translocation or a double inversion (Koller 1935) could produce similar loops, but the interpretation given in Fig. 7 is morphologically more probable; on the other hand, the most important thing discussed here is that one deficiency in one homologous chromosome promotes the formation of a loop and permits the deduction that in Stylonychia mytilus, similarly as in Diptera, early somatic pairing of the two homologues chromosomes occurs. This has been suggested previously by the existence of longitudinal splits in these polytene chromosomes (Jareño 1974). It could be that in Stylonychia mytilus syngen I (now named S. lemnae, Ammermann and Schlegel 1983) the polytene chromosomes are also formed by somatic pairing of the homologues. Some small longitudinal splits that could represent somatic pairing have also been observed in $S$. mytilus syngen I (unpublished results and Jareño 1990).

Summing up, we think that in species of the Stylonychia mytilus complex (Wirnsberger et al. 1986) such as in other species of Hypotricha (Alonso and Pérez, 1967, Spear and Lauth, 1976) polytene chromosomes are formed by the somatic pariring of the two homologues.

\section{Summary}

Polytene chromosomes of Stylonychia mytilus are formed during the development of a macronucleus from a micronucleus after sexual processes such as the conjugation. These chromosomes have many similarities to the polytene chromosomes of the Diptera. This paper deals with the morphological study of a chromosomal loop observed in three different phases of development of polytene chromosomes in Stylonychia mytilus. It was formed in the early stages possibly due to the existence of an inversion in one of the two homologous chromosomes and the subsequent pairing of the homologous tracts. This could answer the question regarding the existence or not of somatic pairing during the development of polytene chromosomes of this species.

\section{Acknowledgements}

I would like to thank Dr. I. B. Raikov for critical reading of the manuscript.

\section{References}

Alonso, P. and Jareño, M. A. 1974. Incorporación de la Uridina-H3 en el esbozo macronuclear de Stylonychia mytilus. Microbiol. Españ. 27: 199-211. 
- and Perez, J. 1965. Giant chromosomes in protozoa. Nature 205: 313.

- and - 1967. Apareamiento somático y politenización en los cromosomas gigantes de ciliados hipotricos. Bol. R. Soc Esp. Hist. Nat. (Biol.) 65: 469-475.

Ammermann, D. 1964. Riesenchromosomen in der Macronucleusanlage des Ciliaten Stylonychia sp Naturwiss. 51: 249.

- 1971. Morphology and development of the macronuclei of ciliates Stylonychia mytilus and Euplotes aediculatus. Chromosoma 33: 209-234.

- and Schlegel 1986. Characterization of two sibling species of the genus Stylonychia (Ciliata, Hypotricha): $S$ mytilus Ehrenberg, 1938 and $S$. lemnae n. sp. I. Morphology and reproductive behavior. J. Protozool. 30: 290-294.

Brito da Cunha A , Morgante, J. S. and Pavan M. C. 1973. Studies on cytology and differentiation in Sciaridae. IV Nuclear and cytoplasmic differentiation in salivary gland of Bradysia elegans (Diptera, Sciaridae). Caryologia 26: 83-100.

Gaude, H. 1981. Zur Funktion des Ciliaten-Riesenchromosomen: Nachweis von RNS-Polymerase in den Polytanen Chromosomen der Makronukleusanlage von Stylonychia mytilus. Arch. Protistenk. 124: 252-258.

Golikowa, M. N. 1964. Polytene chromosomes in the developing macronuclei of ciliates. Tsitologiya 6: 250253.

- and Nilova, V.K. 1985. Autoradiographic investigations of RNA synthesis in the macronucleus of the ciliate Nyctotherus cordiformis at different stages of its life cycle. Acta Protozool. 24: 239-249.

Jareño, M. A. 1974. Etudes sur les chromsomes polytènes de Sytlonychia mytilus: appariement somatique. Protistologica 10: 527-532.

- 1976. Une technique simple pour obtenir des montages permanents de chromosomes polytenes de Stylonychia mytilus. Protistological 12: 593-597.

- 1990. Morphological variations and ectopic pairing of a polytene chromosome of Stylonychia mytilus. Europ. J. Protistol. 26: 110-516.

- and Alonso, P. 1973. Incorporation de l'uridine tritie par les exconjugants de Stylonychia mytilus. Progress in Protozool. Clermont-Ferrand. 199.

--, - and Perez, J. 1972. Identification of some puffed regions in the polytene chromosomes of Stylonychia mytilus. Protistologica 8: 237-243.

Jeon, K. W. (ed.) 1986. Molecular approaches to the study of Protozoa cells. Int. Rev. Cytol. 99: 1-83.

Jha, A. P. and Rhaman, S. M. Z. 1973. On crossing between Drosophila bipectinata and Drosophila malerkotliana. Cytologia 38: $425-436$.

Koller, P. C. 1935. The internal mechanics of the chromosomes. IV Pairing and coiling in salivary gland nuclei of Drosophila. Proc. Roy. Soc. London 118, 371-397.

Raikov, I. B. 1982. The protozoan nucleus. Cell Biol. Monogr. 9: 1-467. Springer Verlag. Wine, New York.

Radzikowski, S. 1967. Nuclear behavior during conjugation and polytene chromosomes in the exconjugants of Chilodonella cucullulus. Bull. Acad. Pol. Sci. 15: 749-751.

Rao, M. V. N. 1968. Macronuclear development in Euplotes woodruffi following conjugation. Exp. Cell. Res. 49: 411-419.

Sapra, G. R. and Dass, C. M. S. 1970. Organization and development of the macronuclear anlage in the Stylonychia notophora. J. Cell Sci. 6: 351-363.

Spear, B. B. and Lauth, M. R. 1976. Polytene chromosomes of Oxytricha: biochemical and morphological changes in a ciliate protozoan. Chromosoma 54:1-13.

Wirnsberger, E., Foissner, W. and Adam, H. 1986. Biometric and morphometric comparison of the sibling species Stylonychia mytilus and $S$. lemnae, including a phylogenetic system for the Oxytrichids (Ciliophora, Hypotrichida). Arch. Protistenkd. 132: 167-185. 